\title{
BMJ Open Impact of cardiovascular comorbidities on COPD Assessment Test (CAT) and its responsiveness to pulmonary rehabilitation in patients with moderate to very severe COPD: protocol of the Chance study
}

\author{
Dionne E Smid, ${ }^{1}$ Sarah Wilke, ${ }^{1}$ Paul W Jones, ${ }^{2}$ Jean W M Muris, ${ }^{3}$ \\ Emiel F M Wouters, ${ }^{1,4}$ Frits M E Franssen, ${ }^{1,4}$ Martijn A Spruit ${ }^{1}$
}

To cite: Smid DE, Wilke S, Jones PW, et al. Impact of cardiovascular comorbidities on COPD Assessment Test (CAT) and its responsiveness to pulmonary rehabilitation in patients with moderate to very severe COPD: protocol of the Chance study. BMJ Open 2015;5:e007536. doi:10.1136/bmjopen-2014007536

- Prepublication history and additional material is available. To view please visit the journal (http://dx.doi.org/ 10.1136/bmjopen-2014007536).

DES and SW are joint first authors.

FMEF and MAS are joint senior authors.

Received 23 December 2014 Revised 16 April 2015 Accepted 29 April 2015

CrossMark

For numbered affiliations see end of article.

Correspondence to Dionne E Smid; dionnesmid@ciro-horn.nl

\section{ABSTRACT}

Introduction: Chronic obstructive pulmonary disease (COPD) is a major cause of morbidity and mortality. Patients with COPD are characterised by a reduced health status, which can be easily assessed by the COPD Assessment Test (CAT). Previous studies show that health status can be worsened by the presence of comorbidities. However, the impact of cardiovascular comorbidities on health status as assessed with CAT is not sufficiently investigated. Therefore, the current study has the following objectives: (1) to study the clinical, (patho)physiological and psychosocial determinants of the CAT, and impact of previously established and/or newly diagnosed cardiovascular comorbidities on health status in tertiary care patients with COPD; (2) to assess the effects of pulmonary rehabilitation on CAT scores in patients with COPD; (3) to develop reference values for the CAT in Dutch elderly patients without COPD; and (4) to validate the CAT in a broad sample of Dutch patients with COPD.

Methods and analysis: The COPD, Health status and Comorbidities (Chance) study is a monocentre study consisting of an observational cross-sectional part and a longitudinal part. Demographic and clinical characteristics will be assessed in primary care, secondary care and tertiary care patients with COPD, and in patients without COPD. To assess health status, the CAT, Clinical COPD Questionnaire (CCQ) and St George's Respiratory Questionnaire (SGRQ) will be used. The longitudinal part consists of a comprehensive pulmonary rehabilitation programme in 500 tertiary care patients. For the cross-sectional part of the study, 150 patients without COPD, 100 primary care patients and 100 secondary care patients will be assessed during a single home visit.

Ethics and dissemination: The Medical Ethical Committee of the Maastricht University Medical Centre+ (MUMC+), Maastricht, the Netherlands (METC 11-3-070), has approved this study. The study has been registered at the Dutch Trial Register (NTR 3416).

\section{BACKGROUND}

Health status in patients with chronic obstructive pulmonary disease (COPD) is impaired irrespective of the degree of airflow limitation. ${ }^{1}$ Therefore, optimising health status is an important goal in COPD management. ${ }^{2}$ Indeed, according to the latest Global initiative for chronic Obstructive Lung Disease (GOLD) document, COPD assessment should include the assessment of health status as an objective in disease diagnosis and follow-up. ${ }^{3}$ Poor health status is multifactorially determined in patients with COPD, as it is associated with higher levels of dyspnoea ${ }^{4}$ reduced exercise capacity, ${ }^{5}$ symptoms of anxiety and depression, ${ }^{6}$ and frequent exacerbations and mortality. ${ }^{7}$ In addition, health status in patients with COPD can be worsened by the presence of comorbidities. ${ }^{8}$ Vanfleteren et at ${ }^{9}$ showed that $97.7 \%$ of all patients with COPD have one or more comorbidities. In European primary care patients with COPD, the presence of $\geq 3$ comorbidities was associated with a worse health status. ${ }^{10}$ Cardiovascular diseases are presumably the most important comorbid conditions in COPD. The risk of cardiovascular morbidity and mortality is twofold to threefold higher in patients with COPD in comparison to an age-matched and gender-matched population without COPD. ${ }^{11}$ Probably due to shared pathophysiological mechanisms, cardiovascular comorbidities often remain unrecognised in patients with COPD. ${ }^{11}$ Rutten et $a l^{12}$ reported a prevalence of $20 \%$ for previously undiagnosed heart failure in primary care patients with COPD. In addition, it was recently shown that echocardiographic 
abnormalities were highly prevalent in patients with COPD at the time of their first hospital admission due to a severe exacerbation. ${ }^{13}$ However, the frequency of echocardiographic abnormalities in patients with COPD referred for pulmonary rehabilitation is not known.

Health status in COPD is often assessed by diseasespecific questionnaires, namely, the St George's Respiratory Questionnaire (SGRQ) ${ }^{14}$ and the Clinical COPD Questionnaire (CCQ). ${ }^{15}$ The SGRQ is reasonably time-consuming to complete, sometimes difficult to understand by patients and has a scoring algorithm that is too complex for routine use in clinical practice. ${ }^{16} \mathrm{In}$ the Netherlands, the CCQ is commonly used in clinical practice. The reliability and validity of the CCQ in patients with COPD have previously been studied. ${ }^{16}$ In addition, a simple eight-item patient-completed questionnaire, the COPD Assessment Test (CAT) was developed some years ago. ${ }^{17}$ However, to a lesser extent, studies investigated the properties of the CAT and associations with clinical, physiological and psychological outcomes in COPD. Additionally, during the period that the current study protocol was designed, few studies about CAT in the Dutch population were published. Therefore, the COPD, Health status and Comorbidities (Chance) study was initiated and the following objectives were formulated:

1. To study the clinical, (patho)physiological and psychosocial determinants of the CAT and impact of previously established and/or new diagnosed cardiovascular comorbidities on health status in tertiary care patients with COPD.

2. To assess the effects of pulmonary rehabilitation on CAT scores in patients with COPD.
3. To develop reference values for the CAT by comparing patients with COPD using elderly Dutch patients without COPD.

4. To validate the CAT in a broad sample of Dutch patients with COPD.

\section{METHODS}

\section{Study design}

The current study is a monocentre, observational study consisting of a cross-sectional part (objectives 1, 3 and 4) and a longitudinal part (objective 2), see figure 1.

\section{Study population}

Patients will be recruited from primary (general practitioners, GPs ), secondary (chest physicians) and tertiary (pulmonary rehabilitation) care. The inclusion of patients started in April 2012. The inclusion of the patients from the tertiary care setting was completed in September 2014. It is expected that the inclusion of the patients without COPD, and patients from the primary and secondary care setting, will be completed in early 2015. Figure 1 shows an overview of the study objectives and study population. In order to study objectives 1 and 2, 500 patients with COPD, referred for clinical assessment and pulmonary rehabilitation to CIRO, Horn, The Netherlands, will be recruited. ${ }^{18}$ In order to examine objective 3 (see figure 1) 150 patients without COPD will be recruited from GPs via the 'Registration Network of Family Practices (RNH) $'{ }^{19}$ Objective 4 (see figure 1) will be studied by assessing 100 patients with COPD from a primary care setting (recruited from GPs via $\mathrm{RNH}$ ) and 100 patients with COPD from secondary

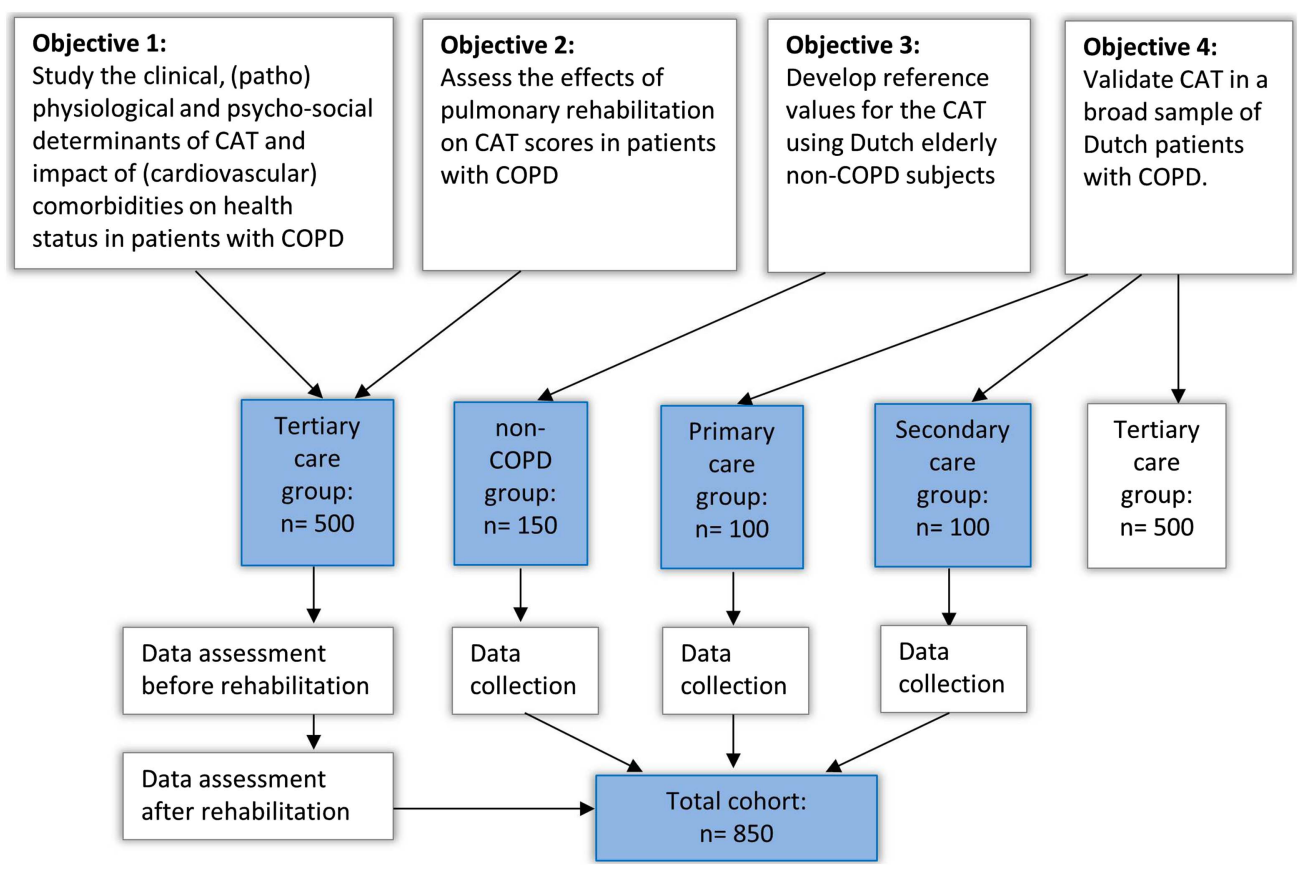

Figure 1 Flow diagram of patient participation and data assessment (CAT, COPD Assessment Test; COPD, chronic obstructive pulmonary disease). 
care settings (partly recruited via RNH and partly at the outpatient pulmonary consultation of Maastricht University Medical Centre (MUMC) Maastricht). Primary care patients are eligible if they are exclusively treated by a GP without being treated by a chest physician or have been treated in tertiary care in the previous 5 years. Secondary care patients are eligible when they are only being treated by a chest physician and have not been treated in tertiary care for the previous 5 years. In addition, 500 patients with COPD from the tertiary care setting will be included for the fourth objective. The 500 tertiary care patients who will be tested for objectives 1 and 2 will be part of the sample for objective 4 . All study procedures will be conducted by CIRO.

\section{Study procedure}

Patients without COPD, primary care patients and part of the secondary care patients will be recruited via RNH. $\mathrm{RNH}$ will provide the contact details of participating GPs. Accordingly, the investigator will contact the responsible GP practices if they are willing to participate. After the GP's approval of collaboration, medical records of the practice are screened using RNH software, according to the eligibility criteria for the study. Following approval of the responsible GP, the investigators from CIRO will send a letter to every eligible patient on behalf of the GP, introducing the research and asking whether the patient wants to participate. In case of patient's consent, a response letter with contact details will be returned to CIRO, enabling the investigator to contact the participant and check the eligibility criteria via phone. If the patient is still eligible and interested, an appointment for a home visit will be scheduled. The remaining secondary care patients will be recruited by chest physicians from an academic hospital (MUMC). During their outpatient pulmonary consultations, the chest physicians will ask the patient if he/she is interested in participating in the study. If the patient is interested, the CIRO investigators will be provided with the contact details, will contact the potential candidate and will possibly schedule an appointment. Patients from primary and secondary care, and patients without COPD, will be visited at their home. A home visit will last approximately $1 \frac{1 / 2}{2}$ to $2 \mathrm{~h}$. If it is not possible to conduct the visit in their home environment, participants will be asked to come to CIRO for $2 \mathrm{~h}$. All patients will be asked to give written informed consent at the beginning of the home visit or at the time of the visit to CIRO. Tertiary care patients will be recruited at CIRO during their prerehabilitation assessment. Eligible patients will be asked if they are willing to participate in the study. After approval and signing the informed consent, required data will be gathered. In these patients, baseline and outcome assessment data will be collected (see figure 1). CIRO is providing a state-of-the-art interdisciplinary pulmonary rehabilitation programme for patients with COPD, in line with the latest American Thoracic Society/European Respiratory Society Statement on Pulmonary Rehabilitation. ${ }^{20}$ Patients are referred for inpatient
(8 weeks) or outpatient (16 weeks) pulmonary rehabilitation based on their pre-rehabilitation assessment. ${ }^{18}$ The pulmonary rehabilitation programme in this study is part of the usual care of these patients at CIRO.

\section{Eligibility criteria}

Patients are eligible if they fulfil the following criteria:

1. Age $40-85$ years.

2. A diagnosis of COPD according to GOLD guidelines. ${ }^{3}$

Patients with COPD from the tertiary care setting also have to fulfil the following criteria:

1. Referral for assessment and pulmonary rehabilitation at CIRO by a chest physician.

Patients without COPD are eligible if they fulfil the following criteria:

1. Age $40-85$ years.

2. Postbronchodilator forced expiratory volume in $1 \mathrm{~s} /$ forced vital capacity $\left(\mathrm{FEV}_{1} / \mathrm{FVC}\right) \geq 70 \%$.

3. Healthy, as judged by the investigator, and determined by medical history and physical examination (specified under the heading 'exclusion criteria for the patients without COPD').

Exclusion criteria for the patients with COPD:

1. A history of asthma, lung cancer, sarcoidosis, tuberculosis, lung fibrosis, cystic fibrosis or any other significant respiratory disease.

2. A moderate or severe exacerbation or pneumonia requiring systemic corticosteroids, antibiotics or hospitalisation during the past 4 weeks.

3. Having undergone lung surgery (eg, lung volume reduction, lung transplantation).

4. Any clinically relevant disease that, in the opinion of the investigator, may influence the results of the study, in other words, diseases influencing health status not related to symptoms of COPD.

5. Malignancy within the past 5 years.

6. For primary care patients: treatment by respiratory physician in secondary or tertiary care.

For secondary care patients: treatment in a tertiary care setting in the previous 5 years.

Exclusion criteria for the patients without COPD:

1. A history of COPD, asthma, lung cancer, sarcoidosis, tuberculosis, lung fibrosis, cystic fibrosis or any other significant respiratory disease, lung surgery in the past.

2. Chronic heart failure in medical history.

3. Any clinically relevant disease that, in the opinion of the investigator, may influence the results of the study, in other words, diseases influencing health status not related to symptoms of COPD.

4. Malignancy within the past 5 years.

\section{Outcomes}

Table 1 provides an overview of the recorded variables for each group. 
Table 1 Outcome measures per healthcare group

\begin{tabular}{|c|c|c|c|c|c|}
\hline Outcomes & Non-COPD & $\begin{array}{l}\text { Primary } \\
\text { care }\end{array}$ & $\begin{array}{l}\text { Secondary } \\
\text { care }\end{array}$ & $\begin{array}{l}\text { Tertiary care } \\
\text { (pre-rehabilitation) }\end{array}$ & $\begin{array}{l}\text { Tertiary care } \\
\text { (postrehabilitation) }\end{array}$ \\
\hline Demographics, including age, gender, height, weight, marital status, ethnic origin & Y & Y & Y & Y & Y \\
\hline Smoking history: current smoking and pack years & Y & Y & $\mathrm{Y}$ & $\mathrm{Y}$ & Y \\
\hline Medical history, including current medication & Y & $\mathrm{Y}$ & $\mathrm{Y}$ & $\mathrm{Y}$ & $\mathrm{N}$ \\
\hline COPD history: number of exacerbations and hospitalisations for COPD ( $<12$ months) & Y & Y & $\mathrm{Y}$ & $\mathrm{Y}$ & Y \\
\hline Use of long-term oxygen or non-invasive ventilation & $\mathrm{Y}$ & Y & Y & Y & $\mathrm{Y}$ \\
\hline $\begin{array}{l}\text { Lung function: postbronchodilator (salbutamol) spirometry measured by a handheld } \\
\text { SpiroPro Viasys }\end{array}$ & $\mathrm{Y}$ & $\mathrm{Y}$ & $\mathrm{Y}$ & $\mathrm{N}$ & $\mathrm{N}$ \\
\hline $\begin{array}{l}\text { Lung function: postbronchodilator (salbutamol) spirometry measured by standardised } \\
\text { equipment from Masterlab, Jaeger, Germany, whole-body plethysmography, diffusing } \\
\text { capacity for carbon monoxide }\end{array}$ & $\mathrm{N}$ & $\mathrm{N}$ & $\mathrm{N}$ & $\mathrm{Y}$ & Y \\
\hline Degree of self-perceived physical and psychological symptoms* & Y & Y & Y & Y & $\mathrm{N}$ \\
\hline Physical examination including vital signs: pulse, blood pressure, saturation & Y & Y & $\mathrm{Y}$ & $\mathrm{Y}$ & Y \\
\hline Charlson comorbidity index 22 & $\mathrm{Y}$ & Y & $\mathrm{Y}$ & $\mathrm{Y}$ & $\mathrm{Y}$ \\
\hline mMRC dyspnoea grading ${ }^{23}$ and NYHA Functional Classification ${ }^{24}$ & $\mathrm{Y}$ & $\mathrm{Y}$ & $\mathrm{Y}$ & $\mathrm{Y}$ & $\mathrm{Y}$ \\
\hline Health status questionnaires: $\mathrm{SGRQ}-\mathrm{C}, \mathrm{CAT}$ and $\mathrm{CCQ}^{25}$ & $\mathrm{Y}$ & $\mathrm{Y}$ & $\mathrm{Y}$ & $\mathrm{Y}$ & $\mathrm{Y}$ \\
\hline Hospital Anxiety and Depression Scale 26 & Y & Y & $\mathrm{Y}$ & $\mathrm{Y}$ & Y \\
\hline Daily physical functioning: timed 'up-and-go' test ${ }^{27}$ & Y & Y & Y & Y & Y \\
\hline Care Dependency Scale 28 & $\mathrm{Y}$ & $\mathrm{Y}$ & $\mathrm{Y}$ & $\mathrm{Y}$ & $\mathrm{Y}$ \\
\hline Coping strategies: Utrecht Coping List ${ }^{37}$ & $\mathrm{~N}$ & $\mathrm{~N}$ & $\mathrm{~N}$ & $\mathrm{Y}$ & Y \\
\hline Body composition: fat-free mass, fat mass using bioelectrical impedance assessment ${ }^{29}$ & $\mathrm{Y}$ & $\mathrm{Y}$ & $\mathrm{Y}$ & $\mathrm{Y}$ & $\mathrm{N}$ \\
\hline Body composition: whole-body/local DEXA scan ${ }^{30}$ & $\mathrm{~N}$ & $\mathrm{~N}$ & $\mathrm{~N}$ & $\mathrm{Y}$ & $\mathrm{Y}$ \\
\hline Systemic inflammation: hsCRP & $\mathrm{N}$ & $\mathrm{N}$ & $\mathrm{N}$ & $\mathrm{Y}$ & Y \\
\hline 6 Minute Walk test $(2 \times \text { at baseline })^{31}$ & $\mathrm{~N}$ & $\mathrm{~N}$ & $\mathrm{~N}$ & $\mathrm{Y}$ & $\mathrm{Y}$ \\
\hline Constant work-rate bicycle test ${ }^{32}$ and cardiopulmonary exercise test & $\mathrm{N}$ & $\mathrm{N}$ & $\mathrm{N}$ & Y & $\mathrm{Y}$ \\
\hline Daily physical activity level using a validated accelerometer ${ }^{33}$ & $\mathrm{~N}$ & $\mathrm{~N}$ & $\mathrm{~N}$ & Y & Y \\
\hline Problematic activities of daily life: Canadian Occupational Performance Measure ${ }^{34}$ & $\mathrm{~N}$ & $\mathrm{~N}$ & $\mathrm{~N}$ & $\mathrm{Y}$ & $\mathrm{Y}$ \\
\hline Lower limb muscle function: peak isokinetic quadriceps strength using a Biodex ${ }^{38}$ & $\mathrm{~N}$ & $\mathrm{~N}$ & $\mathrm{~N}$ & $\mathrm{Y}$ & Y \\
\hline Echocardiography & $\mathrm{N}$ & $\mathrm{N}$ & $\mathrm{N}$ & $\mathrm{Y}$ & $\mathrm{N}$ \\
\hline $\mathrm{ECG}^{39}$ & $\mathrm{~N}$ & $\mathrm{~N}$ & $\mathrm{~N}$ & $\mathrm{Y}$ & $\mathrm{Y}$ \\
\hline NT-proBNP and other cardiovascular markers (to be determined) & $\mathrm{N}$ & $\mathrm{N}$ & $\mathrm{N}$ & $\mathrm{Y}$ & Y \\
\hline Biomarkers metabolic syndrome: ${ }^{40}$ fasting glucose, cholesterol, HDL, LDL, triglycerides & $\mathrm{N}$ & $\mathrm{N}$ & $\mathrm{N}$ & Y & Y \\
\hline
\end{tabular}

*Patient-completed checklist referring to dyspnoea, fatigue, cough, muscle strength, appetite, insomnia, depression, anxiety, panic attacks, pain, mouth soreness, itching, oedema, thirst, muscle

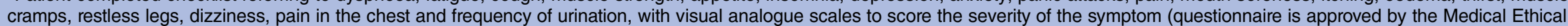
Committee of the Maastricht University Medical Centre, METC 07-3-054).

CAT, COPD Assessment Test; CCQ, Clinical COPD Questionnaire; COPD, chronic obstructive pulmonary disease; DEXA, dual-energy X-ray absorptiometry; HDL, high-density lipoprotein;

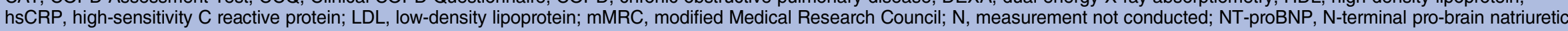
peptide; NYHA, New York Heart Association; SGRQ-C, COPD-specific version of the St. George respiratory questionnaire; Y, measurement conducted. 


\section{Sample size calculation}

The protocol was developed in 2012. At that time, the minimally clinically important difference (MCID) had not yet been established for the CAT. An estimation of the MCID was made to calculate the sample size for the current study. During the study period, the MCID of the CAT was set on two points. ${ }^{35}$ Subsequently, the sample size calculation was adjusted based on the most recent findings (calculated with the program Gxpower 3.1.9). Resulting in a study population of 150 patients without COPD, 100 primary care patients, 100 secondary care patients and 500 tertiary care patients. The full sample size calculation is accessible via the online supplementary material.

\section{Data management and statistics}

Data will be screened for missing values. In order to reduce the number of missing data, a researcher will be present when filling out the questionnaires. When there are missing data in the questionnaires, the missing values will be processed according to the guidelines of the different questionnaires. This will be carried out for every variable and participant. Other missing values will be excluded by list-wise deletion.

All variables will be tested for normality. Descriptive statistics, including means (SD), medians (IQR) and frequencies, will be applied. Continuous variables will be presented as mean $(95 \% \mathrm{CI})$. To answer objective 1, the differences between groups will be assessed with unpaired Student t test. Multiple clinical outcomes will be tested for their association with CAT scores via multiple ordinary least squares (OLS) regression models. For objective 2, an analysis of variance (ANOVA) of repeated measurement will be performed to measure the change in CAT scores and a one-way ANOVA or twotailed paired Student $\mathrm{t}$ test will be used to determine changes in CAT scores following a comprehensive pulmonary rehabilitation programme. To examine objective 3 , the characteristics and the CAT scores of the patients without COPD will be tested for normality with the Kolmogorov-Smirnov test. To validate and look at reference values for the CAT, the upper limit of the $95 \%$ CI of the CAT scores will be determined in the patients without COPD. All scores above this value will be defined as 'an abnormal health status'. For objective 4, differences in the CAT scores and other clinical characteristics between primary care and secondary care, and tertiary care COPD samples, will be assessed by using a one-way ANOVA. Finally, the scores of the CAT between the groups of primary, secondary and tertiary care, and patients without COPD, will be examined. All statistics will be processed using SPSS V.20.0 and GraphPad Prism. A p value of less than 0.05 is considered statistically significant.

\section{Dissemination}

Study data will be stored in the data centre at CIRO. The investigator will ensure that all data in the data centre are accurate and will be responsible for the monitoring of the data collection. Results will be presented at (inter) national conferences and will be submitted for publication in peer-reviewed journals. Participants are given the opportunity to be informed about the results of the study.

\section{DISCUSSION}

The current study is designed to examine the validity and responsiveness of the CAT to pulmonary rehabilitation in a Dutch population. Initially, the clinical, (patho)physiological and psychosocial determinants of the CAT, and impact of cardiovascular comorbidities on health status in tertiary care patients with COPD, will be examined. In addition, reference values for the CAT will be developed by comparing patients with COPD with elderly Dutch patients without COPD. The strengths and limitations of the current study are described below.

\section{Strengths}

In the current literature, most COPD studies focus on patients from secondary or tertiary care. ${ }^{36}$ To our knowledge, this is the first study including patients with COPD treated in primary care as well as patients with COPD treated in secondary and tertiary care. In addition, the current study includes patients without COPD, enabling a comparison between primary, secondary and tertiary care patients and patients without COPD, regarding, for example, health status, mood status and functional status. Consequently, reference values for the CAT in Dutch elderly patients without COPD can be determined. Additionally, the majority of the measurements will be taken with the same devices. This provides a high reliability, despite the fact that the measurements will be carried out at different places. Furthermore, interobserver bias will be minimised, because all measurements in patients without COPD, primary care and secondary care patients will be performed by one researcher. Furthermore, as mentioned before, patients with COPD have a twofold to threefold higher chance of developing cardiovascular morbidity and higher risk of mortality than people without COPD ${ }^{11}$ underscoring the importance of assessing these comorbid conditions carefully. The current study is the first to investigate a wide range of (extra) pulmonary parameters, providing the possibility of studying the individual effect of cardiovascular comorbidities on outcomes, for example, health status. Finally, patients are recruited from eight different GP practices (RNH affiliated), an academic hospital and a pulmonary rehabilitation centre (CIRO), increasing the internal and external validity.

\section{Limitations}

The results of the current study will be subject to several limitations. First, the study sample consists of a convenience sample: possibly in all four healthcare groups the patients with more symptoms, lack of motivation or 
more severe COPD are less willing to participate in the study, which can lead to selection bias. Consequently, outcomes can be more favourable. To limit selection bias as much as possible, every eligible patient without COPD, primary care patient and secondary care patient will be approached by their GP or chest physician, respectively, to participate in the current study. Second, health status may seem a subjective measure. Questionnaires addressing health status usually look at the emotional, psychological and physical effect of a disease. Measuring health status implies quantifying the impact of the illness on health, well-being and daily life, in a standardised and objective manner. According to Jones, the end product does not give a clinical impression, because an impaired health status may express itself differently in each patient. However, these questionnaires make it possible to compare health status in patients with COPD. ${ }^{41}$ Third, spirometry will not be performed with the same devices. The spirometry performed in tertiary patients with COPD will be carried out at CIRO as a part of patients' usual care with standardised spirometer equipment from Masterlab. However, this device is not portable, making it impossible to take on home visits. Therefore, the SpiroPro Viasys will be used to measure lung function in patients without COPD, and primary and secondary care patients. Both devices are valid and reliable instruments, ${ }^{21} 42$ and are currently used in COPD studies. ${ }^{43} 44$ The choice is made to perform only one measurement method per person, to decrease the risk of adverse effects (such as exhaustion). Subsequently, it is important to consider that spirometry is mainly performed to confirm or exclude diagnoses in the different populations. FEV1 or FEV1/FVC are no outcome parameters in the current study. Fourth, comorbidities are extensively assessed in tertiary care. Comprehensive comorbidity assessment is not being undertaken for patients without COPD, primary and secondary care patients with COPD. These groups only completed the Charlson comorbidity index. Finally, measurements in primary and secondary care patients as well as patients without COPD will only be conducted cross-sectionally, not providing the possibility to determine causality.

\section{Clinical consequences}

The current study is very likely to have clinical implications. Initially, it will give more insight in understanding the systemic effects of COPD, especially the impact of cardiovascular comorbidities on health status. By performing echocardiography, we will be able to examine cardiac abnormalities, for example, an impaired systolic left ventricular function, valvular abnormalities or increased right ventricular pressures, in relation to clinical outcomes in COPD. This will enable better monitoring of patients and ensure patient safety during pulmonary rehabilitation. Ultimately, patients at risk can receive more personalised, predictive, preventive and participatory ( $\mathrm{P} 4$ medicine) care, for example, to prevent worsening and/or to optimise health status. ${ }^{45}$ In addition, the current study will examine whether the CAT is a valid measurement to assess health status in Dutch patients, and local reference values for clinical practice will be developed. Moreover, by comparing patients without COPD and primary, secondary and tertiary care patients with COPD, this study will increase our understanding of similarities and differences between the various healthcare categories in the Netherlands.

\section{Conclusion}

To conclude, health status is an important patient-related outcome in COPD. Thus, understanding the validity, responsiveness and clinical determinants of the CAT is essential for the management of patients with this disease. The Chance study will greatly extend the current knowledge on the CAT in patients with COPD and without COPD. In this article, the study protocol is described, and possible strengths and limitations are outlined.

\section{Author affiliations}

${ }^{1}$ Department of Research \& Education, CIRO, Horn, The Netherlands ${ }^{2}$ Division of Clinical Science, St George's University of London, London, UK ${ }^{3}$ Department of Family Medicine, CAPHRI School of Public Health and Primary Care, Maastricht University, Maastricht, The Netherlands ${ }^{4}$ Department of Respiratory Medicine, Maastricht University Medical Centre, Maastricht, The Netherlands

Contributors DES, SW, EFMW, FMEF and MAS contributed to the conception and design, interpretation of the data, writing and critical revision of the manuscript. JWMM contributed to the recruitment of the patients and critical revision of the manuscript. PWJ contributed to interpretation of the data and critical revision of the manuscript. All the authors read and approved the final version.

Funding The CAT study was supported by the Lung Foundation Netherlands (3.4.10.015) and GlaxoSmithKline (SC0115406).

\section{Competing interests None declared}

Patient consent Obtained.

Ethics approval The Medical Ethical Committee of the Maastricht University Medical Centre+ (MUMC+), Maastricht, the Netherlands (METC 11-3-070), has approved this study.

Provenance and peer review Not commissioned; externally peer reviewed.

Open Access This is an Open Access article distributed in accordance with the Creative Commons Attribution Non Commercial (CC BY-NC 4.0) license, which permits others to distribute, remix, adapt, build upon this work noncommercially, and license their derivative works on different terms, provided the original work is properly cited and the use is non-commercial. See: http:// creativecommons.org/licenses/by-nc/4.0/

\section{REFERENCES}

1. Agusti A, Calverley PM, Celli B, et al. Characterisation of COPD heterogeneity in the ECLIPSE cohort. Respir Res 2010;11:122.

2. Vestbo J, Hurd SS, Agusti AG, et al. Global strategy for the diagnosis, management, and prevention of chronic obstructive pulmonary disease: GOLD executive summary. Am J Respir Crit Care Med 2013;187:347-65.

3. Global Strategy for the Diagnosis, Management, and Prevention of Chronic, Obstructive Pulmonary Disease. Updated 2015. [PDF file] http://www.goldcopd.com (updated January 2015.6-3-2015).

4. Hajiro $T$, Nishimura $\mathrm{K}$, Tsukino $\mathrm{M}$, et al. A comparison of the level of dyspnea vs disease severity in indicating the health-related quality of life of patients with COPD. Chest 1999;116:1632-7. 
5. Spruit MA, Watkins ML, Edwards LD, et al. Determinants of poor 6-min walking distance in patients with COPD: the ECLIPSE cohort. Respir Med 2010;104:849-57.

6. Cully JA, Graham DP, Stanley MA, et al. Quality of life in patients with chronic obstructive pulmonary disease and comorbid anxiety or depression. Psychosomatics 2006;47:312-19.

7. Domingo-Salvany A, Lamarca R, Ferrer M, et al. Health-related quality of life and mortality in male patients with chronic obstructive pulmonary disease. Am J Respir Crit Care Med 2002;166:680-5.

8. Sin DD, Wu L, Man SF. The relationship between reduced lung function and cardiovascular mortality: a population-based study and a systematic review of the literature. Chest 2005;127:1952-9.

9. Vanfleteren LE, Spruit MA, Groenen M, et al. Clusters of comorbidities based on validated objective measurements and systemic inflammation in patients with chronic obstructive pulmonary disease. Am J Respir Crit Care Med 2013;187:728-35.

10. Jones PW, Brusselle G, Dal Negro RW, et al. Properties of the COPD assessment test in a cross-sectional European study. Eur Respir J 2011;38:29-35.

11. Huiart L, Ernst $P$, Suissa $S$. Cardiovascular morbidity and mortality in COPD. Chest 2005:128:2640-6.

12. Rutten FH, Cramer MJ, Grobbee DE, et al. Unrecognized heart failure in elderly patients with stable chronic obstructive pulmonary disease. Eur Heart J 2005;26:1887-94.

13. Freixa X, Portillo K, Pare C, et al. Echocardiographic abnormalities in patients with COPD at their first hospital admission. Eur Respir $J$ 2013;41:784-91.

14. Jones PW, Quirk FH, Baveystock CM, et al. A self-complete measure of health status for chronic airflow limitation. The St. George's Respiratory Questionnaire. Am Rev Respir Dis 1992;145:1321-7.

15. van der Molen T, Willemse BW, Schokker S, et al. Development, validity and responsiveness of the Clinical COPD Questionnaire. Health Qual Life Outcomes 2003;1:13.

16. Ringbaek T, Martinez G, Lange P. A comparison of the assessment of quality of life with CAT, CCQ, and SGRQ in COPD patients participating in pulmonary rehabilitation. COPD 2012;9:12-15.

17. Jones PW, Harding G, Berry P, et al. Development and first validation of the COPD Assessment Test. Eur Respir J 2009;34:648-54.

18. Spruit MA, Vanderhoven-Augustin I, Janssen PP, et al. Integration of pulmonary rehabilitation in COPD. Lancet 2008;371:12-13.

19. Metsemakers JF, Hoppener P, Knottnerus JA, et al. Computerized health information in The Netherlands: a registration network of family practices. Br J Gen Pract 1992;42:102-6.

20. Spruit MA, Singh SJ, Garvey C, et al. An official American Thoracic Society/European Respiratory Society statement: key concepts and advances in pulmonary rehabilitation. Am J Respir Crit Care Med 2013:188:e13-64.

21. Munnik $P$, Zanen $P$, Lammers JW. A comparison of lung function equipment with emphasis on interchangeability and methods. Physiol Meas 2006;27:445-55.

22. Charlson M, Szatrowski TP, Peterson J, et al. Validation of a combined comorbidity index. J Clin Epidemiol 1994;47:1245-51.

23. van der Molen T, Miravitlles M, Kocks JW. COPD management: role of symptom assessment in routine clinical practice. Int J Chron Obstruct Pulmon Dis 2013;8:461-71.

24. Janssen DJ, Spruit MA, Uszko-Lencer NH, et al. Symptoms, comorbidities, and health care in advanced chronic obstructive pulmonary disease or chronic heart failure. J Palliat Med 2011;14:735-43.

25. Wilke S, Smid DE, Spruit MA, et al. The 2014 Updated GOLD Strategy: a comparison of the various scenarios. J COPD F 2014;1:212-20.

26. Zigmond AS, Snaith RP. The hospital anxiety and depression scale. Acta Psychiatr Scand 1983;67:361-70.
27. Mesquita R, Janssen DJ, Wouters EF, et al. Within-day test-retest reliability of the Timed Up \& Go test in patients with advanced chronic organ failure. Arch Phys Med Rehabil 2013;94:2131-8.

28. Janssen DJ, Wouters EF, Schols JM, et al. Care dependency independently predicts two-year survival in outpatients with advanced chronic organ failure. J Am Med Dir Assoc 2013;14:194-8.

29. Schols AM, Wouters EF, Soeters PB, et al. Body composition by bioelectrical-impedance analysis compared with deuterium dilution and skinfold anthropometry in patients with chronic obstructive pulmonary disease. Am J Clin Nutr 1991;53:421-4.

30. Rutten EP, Spruit MA, Wouters EF. Critical view on diagnosing muscle wasting by single-frequency bio-electrical impedance in COPD. Respiratory medicine 2006;27:445-55.

31. Hernandes NA, Wouters EF, Meijer K, et al. Reproducibility of 6-minute walking test in patients with COPD. Eur Resp J 2011;38:261-7.

32. van't Hul A, Gosselink R, Kwakkel G. Constant-load cycle endurance performance: test-retest reliability and validity in patients with COPD. J Cardiopulm Rehabil 2003;23:143-50.

33. Pitta F, Troosters T, Probst VS, et al. Quantifying physical activity in daily life with questionnaires and motion sensors in COPD. Eur Resp J 2006;27:1040-55

34. Nieuwenhuizen MG, de Groot S, Janssen TW, et al. Canadian Occupational Performance Measure performance scale: Validity and responsiveness in chronic pain. Journal of rehabilitation research and development 2014;51:727-46.

35. Kon SS, Canavan JL, Jones SE, et al. Minimum clinically important difference for the COPD Assessment Test: a prospective analysis. Lancet Respir Med 2014;2:195-203.

36. Herland K, Akselsen JP, Skjonsberg $\mathrm{OH}$, et al. How representative are clinical study patients with asthma or COPD for a larger "real life" population of patients with obstructive lung disease? Respir Med 2005;99:11-19.

37. Stoilkova A, Wouters EF, Spruit MA et al. The relationship between coping styles and clinical outcomes in patients with COPD entering pulmonary rehabilitation. Copd 2013;10:316-23.

38. Franssen FM, Broekhuizen R, Janssen PP, et al. Limb muscle dysfunction in COPD: effects of muscle wasting and exercise training. Medicine and science in sports and exercise 2005;37:2-9.

39. Sin DD, Man SF. Why are patients with chronic obstructive pulmonary disease at increased risk of cardiovascular diseases? The potential role of systemic inflammation in chronic obstructive pulmonary disease. Circulation 2003;107:1514-19.

40. Alberti KG, Eckel RH, Grundy SM, et al. Harmonizing the metabolic syndrome: a joint interim statement of the International Diabetes Federation Task Force on Epidemiology and Prevention; National Heart, Lung, and Blood Institute; American Heart Association; World Heart Federation; International Atherosclerosis Society; and International Association for the Study of Obesity. Circulation 2009;120:1640-5

41. Jones PW. Health status measurement in chronic obstructive pulmonary disease. Thorax 2001;56:880-7.

42. De Soomer K, Claus L, Heyndrickx R, et al. Office spirometry: (un) reliability of some handheld spirometers. European Respiratory Society International Congress; Bercelona, Spain, 2013.

43. Houben $\mathrm{CH}$, Spruit MA, Wouters EF, et al. A randomised controlled trial on the efficacy of advance care planning on the quality of end-of-life care and communication in patients with COPD: the research protocol. BMJ Open 2014;4:e004465.

44. Nakken N, Janssen DJA, van den Bogaart EHA, et al. An observational, longitudinal study on the home environment of people with chronic obstructive pulmonary disease: the research protocol of the Home Sweet Home study. BMJ Open 2014;4:e006098.

45. Auffray C, Charron D, Hood L. Predictive, preventive, personalized, participatory medicine: back to the future. Genome Med 2010;2:57. 